\title{
Flexible Electronics for Efficient Nerve Stimulation
}

\author{
Sefa Şimşek ${ }^{1 *}$, Zafer Soybaş ${ }^{2 *}$, Yakup Tatar ${ }^{3}$, Merve Marçalı ${ }^{4}$, Çağlar Elbüken ${ }^{5}$, Bahattin Aydoğdu ${ }^{6}$, \\ Rohat Melik ${ }^{7+}$ \\ ${ }^{1,2,7}$ Department of Electrical-Electronics Engineering, TOBB University of Economics and Technology \\ 06530 Ankara, Turkey \\ s.simsek@etu.edu.tr; zsoybas@etu.edu.tr; rmelik@etu.edu.tr \\ ${ }^{3}$ Department of Physiology, Faculty of Medicine, University of Ankara, 06100 Ankara, Turkey \\ yakuptatar84@gmail.com \\ ${ }^{4,5}$ Institute of Materials Science and Nanotechnology National Nanotechnology Research Center (UNAM), \\ Bilkent University, 06800 Ankara, Turkey \\ merve.marcali@gmail.com, elbuken@unam.bilkent.edu.tr \\ ${ }^{6}$ Department of Pediatric Surgery and Pediatric Urology, Medical Faculty of Dicle University, 21280 Diyarbakir, Turkey \\ bahattinaydogdu@hotmail.com \\ *These authors contributed equally to this work, ${ }^{+}$Corresponding author
}

\section{Extended Abstract}

Conventional functional electrical stimulation is a currently available treatment method for neurological disorders or spinal cord injury [1]. This conventional electrical stimulation results in unwanted nerve signals and temporary or permanent damage on tissues because of the requirement of high current values [2]. In order to prevent these problems, we propose to reduce the threshold value for stimulation which is the minimum required current value to stimulate the nerve. In this study, stimulation current was applied with microfabricated planar and flexible electrodes to reduce the threshold whereas the conventional functional electrical stimulation is implemented with conventional cable. The planar electrode was fabricated with the standard lithography techniques by coating gold on a glass wafer. On the other hand, in order to fabricate the flexible electrode, we used kapton tape as a substrate. These microfabrication methods are provided by the developments of the nanotechnology and we applied these techniques for biomedical applications.

The threshold values for these three electrodes; conventional cable, microfabricated planar electrode and flexible electrode were measured in-situ along the sciatic nerve of a frog. All the animal experiments were performed in pursuance of the ethical protocols. The sciatic nerve which is integrated with the gastrocnemius muscle, was dissected from the frog. To measure the threshold value, the stimulation current was applied by the electrode, and the resulting force on the gastrocnemius muscle was recorded via force transducer [3]. The threshold values for the three types of electrodes were detected by observing whether there is a muscle contraction or not as a result of the stimulation current applied. The microfabricated planar electrode reduced the threshold values $50 \%$ compared to the conventional functional stimulation due to increase in contact area between the nerve and the electrode. Furthermore, the flexible electrode reduced the threshold values more by $20 \%$ in comparison to a planar electrode. This improvement was achieved by increasing the contact area more, through surrounding the nerve by flexible electrode.

In conclusion, since the flexible electrode provides efficient contact area in a small device, it can be implanted into a body. Moreover, it is the most harmless way to stimulate the nerve by decreasing the stimulation current applied. Hence, the flexible electrode can be used for implantable neuroprosthetic devices for treatment of neural disease or injury.

This work is supported by The Scientific and Technological Research Council of Turkey (TÜBİTAK) 113 S081 grant.

\section{References}

[1] K. D. Anderson, "Targeting recovery: priorities of the spinal cord-injured population," J. Neurotrauma, vol. 21, pp. 1371-1383, 2004. 
[2] M. A. Nitsche, D. Liebetanz, N. Lang, A. Antal, F. Tergau, and W. Paulus, "Safety criteria for transcranial direct current stimulation (tDCS) in humans.," Clinical neurophysiology: official journal of the International Federation of Clinical Neurophysiology, vol. 114. pp. 2220-2222; author reply 2222-2223, 2003.

[3] J. V Basmajian and C. J. De Luca, "Muscles alive," Baltimore, 1985. 